\title{
Exosomes in Post-Ischemic Brain
}

\author{
Ryszard Pluta ${ }^{1}$ - Mirosław Jabłoński \\ ${ }^{1}$ Laboratory of Ischemic and Neurodegenerative Brain Research, Mossakowski Medical \\ Research Institute, Polish Academy of Sciences, Warsaw, Poland; ${ }^{2}$ Department of \\ Rehabilitation and Orthopaedics, Medical University of Lublin, Lublin, Poland
}

Author for correspondence: Ryszard Pluta, Laboratory of Ischemic and Neurodegenerative Brain Research, Mossakowski Medical Research Institute, Polish Academy of Sciences, Warsaw, Poland. Email: pluta@imdik.pan.pl

Cite this chapter as: Pluta R, Jabłoński M. Exosomes in Post-Ischemic Brain. In: Pluta R, editor. Cerebral Ischemia. Brisbane (AU): Exon Publications; 2021. Online first Aug 31.

Doi: https://doi.org/10.36255/exonpublications.cerebralischemia.2021.exosomes

\begin{abstract}
Ischemic stroke is a destructive vascular disease that carries the risk of high mortality, disability, and eventually the development of full-blown dementia. Despite the continuous development of new prognostic methods, the prediction of ischemic sequelae and early and late prognosis of stroke is still much easier said than to apply in practice. Cell-to-cell communication between neuronal, glial, and vascular cells are essential for normal functioning of the brain, and in cerebral ischemia, this communication is interrupted. New research has demonstrated the important role of exosomes in cell-to-cell communication via microRNA transfer, playing an integral role in multicellular crosstalk. Following a stroke, harmful and/or beneficial microRNAs are released into the circulation, significantly affecting the severity and prognosis of a stroke. This chapter provides an overview of the current literature on the possible harmful and beneficial roles of cargo derived from exosomes in ischemic stroke. A snapshot of experimental evidence for the role of exosome-derived microRNAs in ischemic stroke followed by clinical studies exploring the diagnostic and prognostic potential of exosomes in stroke patients are presented. Finally, the promises and pitfalls along with future directions are discussed.
\end{abstract}

In: Cerebral Ischemia. Pluta R (Editor). Exon Publications, Brisbane, Australia. ISBN: 978-0-6450017-9-2; Doi: https://doi.org/10.36255/exonpublications. cerebralischemia.2021

Copyright: The Authors.

License: This open access article is licenced under Creative Commons Attribution-NonCommercial 4.0 International (CC BY-NC 4.0) https://creativecommons.org/licenses/by-nc/4.0/ 
Keywords: Alzheimer's disease; exosomes; extracellular vesicles; ischemic stroke; microRNAs

\section{INTRODUCTION}

Human ischemic stroke is a leading cause of morbidity, mortality, irreversible dementia, progressive increase in healthcare costs, and a burden on families and caregivers around the world. Early diagnosis of cerebral ischemia, and the prediction of ischemic sequelae and prognosis are often difficult. Currently, the treatment for ischemic stroke is thrombolysis and/or thrombectomy, but a strict time window is required for these procedures (1). Nowadays, the diagnosis of acute ischemic stroke is based mainly on advanced neuroimaging techniques, including computed tomography and magnetic resonance imaging, but it should be emphasized that the use of these techniques has numerous limitations. Computed tomography is not sensitive enough in the very early stages of a cerebral infarction, because $40-50 \%$ of all acute ischemic stroke cases show no abnormalities on admission (2). Magnetic resonance imaging has also some disadvantages in its application, such as limited availability (3), the high cost of scanners, and the long duration of the procedure (4). Therefore, a faster and simpler tool is essential for the rapid diagnosis of acute ischemic stroke.

It is known that neurons, neuroglia, and cells associated with cerebral vessels are involved in the development of pathological processes after ischemic stroke, with each cell type contributing differently to the post-ischemic mechanisms of development of neurodegenerative changes in the brain. Exosomes are newly defined structures of interaction between these cells due to their involvement in signaling and intercellular communication. The exosome load, i.e., the microRNA, which is single-stranded non-coding RNA, can be released into the systemic circulation, indicating that it may be a promising diagnostic marker due to its easy detection, serum stability, and cell-specific expression patterns (5). It has been shown that microRNAs may be associated with the mechanisms underlying the occurrence and development of post-stroke neuropathology, including neuronal apoptosis, oxidative stress, and the occurrence of neuroinflammation $(6,7)$. Previous studies have found abnormal accumulation of microRNAs in patients with acute ischemic stroke, suggesting a potential diagnostic value of the microRNA $(8,9)$. It should be emphasized that inadequate sample size, inconsistent subject matter or different microRNA detection techniques lead to significant discrepancies between studies as well as inconsistent results (8-10). Nevertheless, the data suggest that exosomes, which are 30-150 nm vesicles, may be useful therapeutic, diagnostic, and prognostic biomarkers in ischemic stroke (11). Exosomes, microvesicles $(10-1000 \mathrm{~nm})$, and apoptotic bodies $(50-5000 \mathrm{~nm})$ are the three important subtypes of extracellular vesicles (12). Exosome biogenesis involves four stages: initiation, endocytosis, development of multivesicular body, and release through the joining of multivesicular bodies with the cell membrane (13). After being released from source cells, exosomes interact through endocytosis, fusion, or ligand-receptor interactions with target cells (14). Secreted by most cells, exosomes contribute to intercellular signaling through cell-to-cell communication in a variety of brain diseases including ischemic stroke $(15,16)$. They can 
also cross the blood-brain barrier and affect the exchange between the brain and the systemic circulation (17). Their water core and lipid bilayer envelope enable the transport of various substances, such as nucleic acids, lipids, and proteins in an autocrine or paracrine manner (18). Proteins that are exclusively present in exosomes, such as certain heat shock proteins, proteins involved in membrane transport and fusion, and tetraspanins, or that are involved in the biogenesis of a multi-vesicle body, or that associated with phospholipases and lipids, are considered as exosome biomarkers (19). Exosomes are abundant in body fluids such as serum, urine, breast milk, cerebrospinal fluid, amniotic fluid, nasal discharge, saliva, semen, synovial fluid, bile, watery fluid, and fluids produced during development various pathologies $(20,21)$.

Valadi et al. were the first to demonstrate exosome-mediated transfer of microRNA as a new way of exchanging genetic material between cells (22). Exosomes are freely available, have different expression patterns, are more stable, and act as nanocarriers that transmit microRNA and siRNA in brain tissue. In addition, the structure and function of the brain can be directly influenced by exosomal microRNAs that are transmitted between normal and damaged parental neurons (14).

Growing evidence points to the crucial role of functional interactions between neurons, microglia, astrocytes, oligodendrocytes, vascular pericytes, extracellular matrix, endothelial cells, and vascular smooth muscle cells that work to maintain homeostasis in the brain microenvironment after a stroke (23-25). Following ischemia, these cells coordinate a response to restore adequate cerebral blood flow, thus reducing neuronal damage and death (26). Activation of these cells triggers transfer of markers to the plasma, extracellular space, and cerebrospinal fluid that are probable indicators of the state of the brain (26). Moreover, the complex interactions between various cells and factors integrate vascular repair processes, response to neuroinflammation, control of the blood-brain barrier, and the survival of various cells in the post-stroke period (27). Release of exosomes from all the above cells has been reported, and some exosomes release neuroprotective factors after ischemia $(28,29)$.

The multifactorial development of ischemic brain injury after ischemic stroke is not only a potential source of identifying diagnostic markers but also for discovering new therapy protocols. This chapter highlights the roles of exosomes in ischemic brain injury. An overview of our current knowledge on the topic, followed by the diagnostic and prognostic role of exosomes in clinical trials involving patients after stroke are presented. A better understanding of the role of exosomes in post-ischemic neurodegeneration could contribute to faster stroke diagnosis, predict stroke outcomes and prognosis, help develop new therapies, improve patient care, and reduce healthcare costs (30).

\section{EXOSOMES IN THE BRAINS OF PATIENTS AFTER ISCHEMIC STROKE}

Numerous studies have assessed the diagnostic and prognostic role of exosomes and their cargo in patients after acute ischemic stroke $(11,31,32)$. One of the most common contents tested in exosomes is microRNAs, which play a distinct 
role in different molecular pathways involved in stroke. Plasma levels of exosomal microRNA-9, $-15 \mathrm{a}$, and -124 are elevated in ischemic stroke patients, and correlate with infarct volume and blood interleukin-6 concentration $(9,31)$. Thus, mircoRNAs may be promising markers for assessing the degree of brain damage caused by ischemic injury (31). Another exosomal microRNA that is elevated in ischemic stroke patients and correlates with stroke severity and poor short-term prognosis is microRNA-223 (32). Exosomal levels of microRNA-134 are significantly increased in patients within 24 hours of ischemic stroke, and these levels are associated with infarct volume, poorer post-stroke prognosis, increased blood levels of interleukin-6, and serum high-sensitivity C-reactive protein (33). On the other hand, blood levels of exosomal microRNA-152-3p were remarkably lower in ischemic stroke patients. In addition, the lowest levels of microRNA-152-3p were observed in cases with large artery atherosclerosis, and these levels were lower in the acute phase of stroke than in the chronic phase of stroke (34). In summary, microRNAs 9, 124, 134, 152-3p, and 223 are associated with stroke severity; microRNAs 134 and 223 with poor prognosis; microRNAs 9, 124 and 134 with infarct volume; and microRNAs 9, 124 and 134 with interleukin-6 level increase. Two other microRNAs that are present in the exosomes of ischemic stroke patients are 21-5p and 30-5p. These microRNAs are associated with apoptosis that distinguish the acute phase of ischemic stroke from the subacute phase and the recovery period (35). In addition, it has been shown that exosomal microRNA-422a and microRNA-125b-2-3p in the blood can be diagnostic biomarkers in stroke patients, and that the combined use of these two microRNAs can be effective in determining the severity of stroke (36). The progression of asymptomatic carotid artery stenosis with more than $50 \%$ lumen stenosis is a potential risk factor for stroke or transient ischemic attacks of the brain. Clinical studies confirmed significantly higher expression of exosomal microRNA-199b-3p, -27b-3p, -130a-3p, -221-3p and -24-3p in these patients (37). It has also been reported that, in addition to microRNAs, exosomes released into the bloodstream after stroke contain pro-inflammatory proteins, as well as $\mathrm{C}$ reactive protein (38).

\section{EXOSOMES FROM NEURONS}

Neurons release exosomes from their somatodendritic compartments to modulate transsynaptic communication, local synaptic plasticity, post-stroke tissue remodeling and regeneration (29). MicroRNA-181c-3p from cortical neurons has been shown to inhibit neuroinflammation in rats with post-ischemic brain injury (39). The beneficial effect is explained by the reduction of the CXCL1 chemokine in astrocytes, a target gene for microRNA-181c-3p that was suppressed by this microRNA in astrocytes (39). In addition, microRNA-98, derived from the exosomes of neurons, acts as an endogenous post-ischemic protective factor by inhibiting microglial phagocytosis and thus mitigating ischemiainduced neuronal death (40). Moreover, neurons have been shown to secrete microRNA-132 from exosomes into endothelial cells, which regulates the integrity of cerebral vessels (41). 


\section{EXOSOMES FROM ASTROCYTES}

Astrocyte-derived exosomes have been shown to attenuate ischemic damage and inhibit apoptosis by suppressing autophagy in neurons (42). Further studies showed the role of exosome-derived microRNA-190b in the inhibition of the gene related to autophagy (43). Subsequently, circSHOC2 derived from ischemic preconditioned astrocytes exosomes was shown to regulate neuron autophagy and apoptosis via the microRNA-7670-3p/SIRTl axis (44). Other microRNAs derived from astrocyte exosomes with similar properties include microRNA-361 and microRNA-34c (45). Both of these microRNAs inhibit cell apoptosis in rats following ischemic brain injury: microRNA-361 regulating AMP-activated protein kinase/mammalian target of the rapamycin signaling pathway and cathepsin B (45) while microRNA-34c is neuroprotective against ischemic brain injury by the Toll-like receptor 7 and the nuclear factor-kappa B/mitogen-activated protein kinase pathways (46). In addition, it has been shown that astrocyte-derived exosomes treated with a semaphorin 3A inhibitor suppress the activation of astrocytes and their microRNA-30c-2-3p and microRNA-184-5p leading to improved function after ischemic stroke in rats (47). Furthermore, synapsin 1 carrying glycoprotein has been found to act as oligomannose which, by binding lectin, promotes neuronal survival when released by astrocyte-derived exosomes during ischemia and influences the interaction between neurons and neuroglia (48). It has been found that the improvement in neuron survival by astrocyte-derived exosomes during post-ischemic injury is also dependent on prion protein (49). Furthermore, microRNA-92b-3p, transported by exosomes to neurons, protects post-ischemic neurons from damage due to oxygen and glucose deficiency, and apoptosis (50).

\section{EXOSOMES FROM MICROGLIA}

It has been shown that microRNA-124, derived from microglia M2 exosomes, has a neuroprotective effect by limiting apoptosis, thus promoting the survival and activity of neuronal cells (39). Moreover, three days after reversible focal cerebral ischemia, exosomal microRNA derived from microglia M2 was found to be elevated in ischemic penumbra (39). M2 microglia-derived exosomes also reduce glial scar development and improve post-ischemic regeneration via the microRNA-124/STAT3 pathway and inhibit astrocyte migration and proliferation. Moreover, this exosomal microRNA has been suggested to be involved in the transition of the astrocyte to the neural progenitor cell via increasing Sox2 and decreasing Notchl expression (51). IL-4-polarized microglial cells have been found to promote post-ischemic angiogenesis by enhancing endothelial cell tube development via the secretion of exosomes containing microRNA-26a (52). A recent study has shown that microglial vesicles improve stroke recovery by preventing immune cell senescence and promoting oligodendrogenesis (53). These exosomes are likely to have a beneficial effect on the maturation of oligodendrocyte precursor cells through the tumor necrosis factor- $\alpha$ /tumor necrosis factor receptor 2 axis (53). 


\section{EXOSOMES FROM OLIGODENDROCYTES}

Exosomes from oligodendrocytes promote neuronal survival through gene regulation and signal transduction in in vitro cerebral ischemia (54). These exosomes carry antioxidant enzymes such as superoxide dismutase 1 and catalase into neuronal cells, thereby increasing their tolerance to oxidative stress (54). Moreover, they stimulate survival mechanisms by regulating extracellular signal-regulated protein kinases 1 and 2 and Akt axis (54).

\section{EXOSOMES FROM ENDOTHELIAL CELLS}

Vascular endothelial exosomes have been found to be essential for the protection of neural stem cells in post-ischemic brain injury (55). The above study showed that the injection of exosomes derived from endothelial cells into the ischemic brain promotes neurogenesis by activating neuronal progenitor cell proliferation and migration into the infarct area, reducing infarct volume, and improving neurological outcomes in rats (55). Exosomes derived from brain endothelial cells induce neuroplasticity, function of synapses, and ultimately improve motor regeneration following ischemic brain injury (56). MicroRNA126-3p influences neuroplasticity in the brain by increasing neurite outgrowth and protecting neurons from injury and apoptosis (56). Another study found that exosomes derived from ischemic brain endothelial cells promote the growth of axons of cortical neurons by modulating microRNAs 19a, 27a, 195 and 298, and by influencing axon inhibitory proteins such as phosphatase, semaphorin $6 \mathrm{~A}$ and tension, and Ras homolog family member A (57). Interestingly, exosomes derived from ischemic endothelial cells have a strong influence in mediating axonal plasticity and homeostasis (57).

Endothelial cell-derived exosomes display neuroprotective effects after cerebral ischemia in type 2 diabetic mice by increasing the number of microvessels, axons and myelin density in the ischemic border zone, as well as polarization of M2 macrophages, which improved neurological and cognitive outcomes possibly mediated by microRNA-126 (58). Another study confirmed the above observations that microRNA-126 from exosomes derived from endothelial progenitor cells reduce infarct volume, maintain normal cerebral blood flow and microcirculation, and improve neurological outcomes by increasing angiogenesis and neurogenesis by lowering caspase-3 levels and increasing vascular regulation of endothelial growth factor receptor 2 (59). In addition, it was found that moderate exercise has a beneficial effect on circulating exosomes derived from endothelial progenitor cells rich in microRNA-126, which have a protective effect on post-ischemic brain damage (60). It was also shown that exosomes derived from femoral artery endothelial cells had a protective effect on neurons after ischemia-reperfusion brain injury (61). 


\section{CONCLUSION}

Neurons, neuroglia, and endothelial cells have been found to influence overall brain function following ischemia. Exosomes derived from these cells and their behavior are newly described processes of interaction between these cells due to their involvement in signaling and cell-to-cell communication. Many studies talk about the role of exosomes and the microRNAs secreted by them (Figure 1); however, these experimental results are not supported by relevant clinical evaluation. Human ischemic stroke is a leading cause of morbidity and mortality worldwide. Despite advances in understanding the underlying pathophysiological mechanisms of stroke, resumption of circulation is the

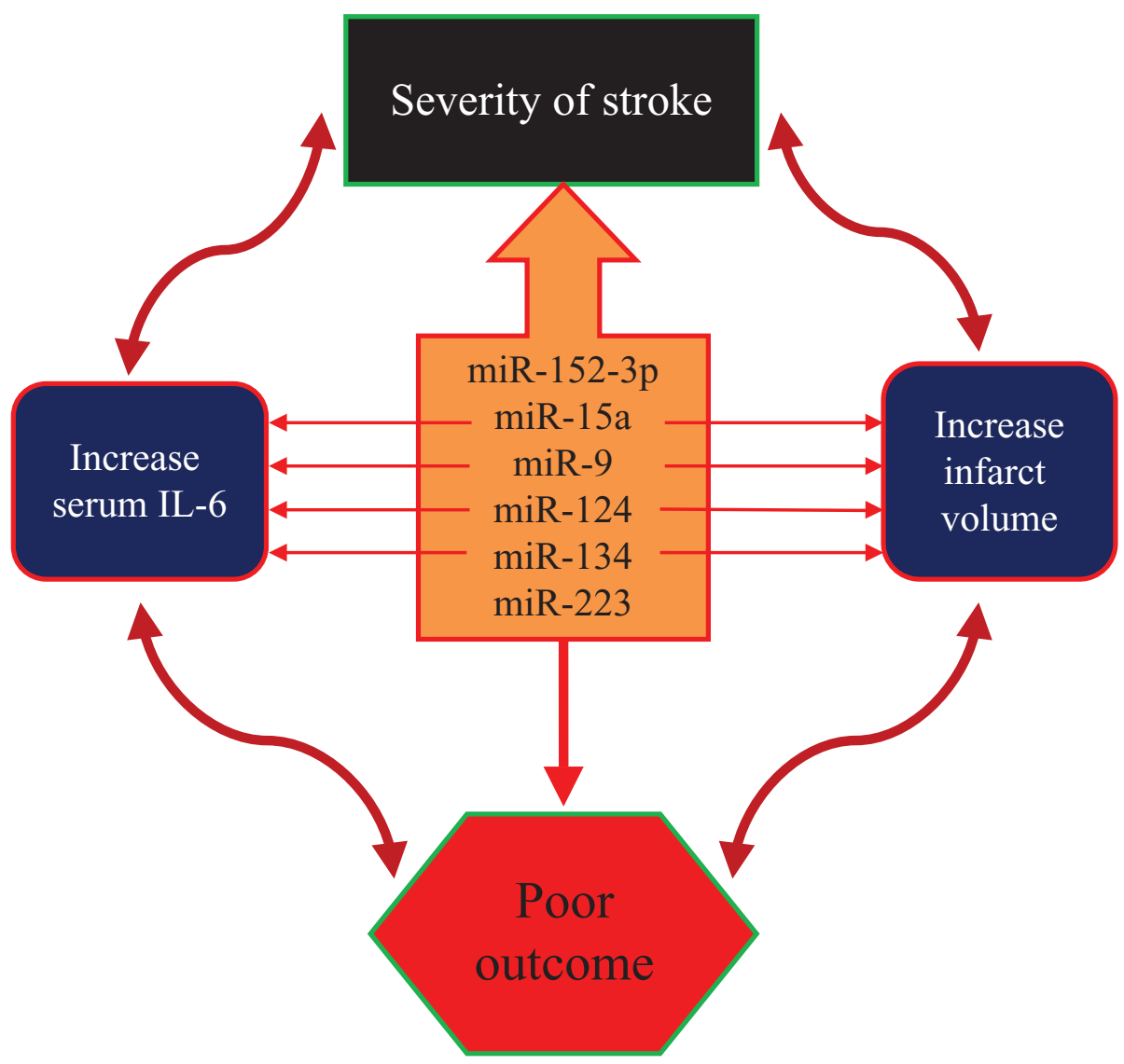

Figure 1. Exosome cargo as a possible prognostic factor of poor prognosis after cerebral ischemia in humans. IL-6-interleukin 6; miR-microRNA. 
only immediate treatment choice for patients with this condition. This indicates a good reason for the clinical need for further research to develop new treatments in this field.

Recent advances in experimental approaches to drug discovery, including high-throughput screening (62) and computer-aided drug design (63), may provide new insight into the identification and validation of potential therapeutic targets for various neurodegenerative diseases, including ischemic stroke. Moreover, these techniques could also serve as the basis for targeted exosome validation in future research. In addition to the above, in vitro models can help improve our understanding of the complex interactions between various structures and elements of brain tissue following an ischemic stroke. Organ-on-chip microfluidic models, such as the perfused blood-brain barrier on-chip (64) and the human brain microvessel on-chip (65), have shown to be suitable for advanced imaging of different phenomena, making them useful in studying the mechanisms of molecular transport involved in transcytosis of nanoparticles or biological agents across the blood-brain barrier. These platforms allow real-time monitoring of permeability changes, thus offering the possibility to study exosome release and penetration during and post-ischemic cerebral ischemia as well as ischemiainduced neuroinflammation. In addition, 3D cultures and organoids are also capable of mimicking blood-brain barrier dysfunction and have the potential to be used in modeling cerebral ischemia and designing therapies (66). Recent preclinical evidence has emerged that brain organoids transplant may be an effective intervention in the treatment of ischemic stroke (67).

We have known for a long time that early diagnosis of stroke and prognosis of post-stroke are often amazing challenges. Cerebral ischemia is a heterogeneous process that cannot be characterized by a single marker. Consequently, diagnostic panels should be composed of multiple biomarkers representing various pathophysiological molecular processes, including neuroinflammatory response, damage to the blood-brain barrier and brain edema, oxidative stress, and necrotic and apoptotic neuronal death (68). A better understanding of the physiological and pathological aspects of exosomes and their cargos will contribute to the precise diagnosis of ischemic stroke, the prediction of its consequences and treatment, and thus to the improvement of patient care and treatment outcomes. In the future, comprehensive stroke diagnostic protocols should include not only clinical and neuroradiological symptoms, but also biomarkers representative of all structures and components of brain tissue. Ischemic stroke in developing and developed countries continues to be an increasing health problem that affects millions of people worldwide.

Despite tremendous progress in ischemic stroke research in understanding the molecular processes of early and late sequelae, we still lack drugs or measures to delay or prevent progression, especially post-stroke, and we have still not identified the biomarkers needed for early detection of this condition. A significant breakthrough in the development of such biomarkers is the discovery that microRNAs connect the missing link between cell changes and disease progression. There are many questions about microRNAs in cerebral ischemia that need to be answered, including which specific microRNAs are involved in the cellular changes associated with the pathogenesis and progression of stroke; which specific 
microRNAs are involved in cellular changes associated with other neurodegenerative disorders and aging; can stroke be prevented, delayed, or arrested through strategic alteration of microRNA expression; and whether and how serum and imaging tests can be developed that will focus on identifying microRNA changes that are responsible for the onset and progression of stroke. Comprehensive microRNA studies are urgently needed to help develop microRNA-based diagnostic tools. At present, because clinical examination may not be decisive and neuroimaging may not be feasible in some cases, non-invasive blood markers are essential in acute ischemic stroke diagnosis. Currently, no commonly recognized markers are available for routine use in the diagnosis, differentiation or stratification of acute stroke risk and its sequelae (69). Thus, microRNAs that are abundantly present in the brain and involved in various neuropathological cellular processes may act as prospective markers that reflect the state and situation of the brain following ischemia (Figure 1) (70). Although many studies have found abnormal levels of microRNAs in acute ischemic stroke patients, a systematic review and meta-analysis is needed to collect and synthesize the available data and thus answer some of the questions. This should be done in addition to many meta-analyzes to comprehensively analyze and summarize the evidence on the influence of circulating microRNAs in the diagnosis and especially in the assessment of the consequences of ischemic stroke. It is expected that the results of these studies will provide clinical evidence as well as the possibilities and future directions of diagnosing and predicting the consequences of ischemic stroke in humans (10).

Also, research suggests that exosomes can be engineered to modify the microRNA content to obtain therapeutic benefits $(71,72)$. For that reason, research to characterize and optimize the content of exosomes is necessary. Moreover, it is necessary to define the principles of safety of exosome therapy, standardize their isolation, optimize the dose or volume of administration, and determine the routes and frequency of administration during therapeutic application. Optimizing the treatment protocols, procedures, and patient responses, which may vary between with old and young patients, as well as between men and women, is necessary. These require further pre-clinical and clinical research. These investigations will be crucial in translating exosome-based treatments into a clinic for treating the aftermath of ischemic stroke.

Acknowledgment: The authors acknowledge the financial support from the Mossakowski Medical Research Institute, Polish Academy of Sciences, Warsaw, Poland (T3-RP).

Conflict of Interest: The authors declare no potential conflicts of interest with respect to research, authorship, and/or publication of this chapter.

Copyright and Permission Statement: To the best of our knowledge, the materials included in this chapter do not violate copyright laws. All original sources have been appropriately acknowledged and/or referenced. Where relevant, appropriate permissions have been obtained from the original copyright holder(s). 


\section{REFERENCES}

1. Xin M, Feng J, Hao Y, You J, Wang X, Yin X, et al. Cyclic adenosine monophosphate in acute ischemic stroke: some to update, more to explore. J Neurol Sci 2020; 413:116775. https://doi.org/10.1016/j. jns.2020.116775

2. Gomolka RS, Chrzan RM, Urbanik A, Kazmierski R, Grzanka AD, Nowinski WL. Quantification of image contrast of infarcts on computed tomography scans. Neuroradiol J 2017;1:15-22. https://doi. org/10.1177/1971400916678226

3. Khaing M, Saw YM, Than TM, Mon AM, Cho SM, Saw TN, et al. Geographic distribution and utilisation of CT and MRI services at public hospitals in Myanmar. BMC Health Serv Res 2020;1:742. https://doi.org/10.1186/s12913-020-05610-x

4. Bornert P, Norris DG. A half-century of innovation in technology preparing MRI for the 21st century. Br J Radiol 2020;1111:20200113. https://doi.org/10.1259/bjr.20200113

5. Hiam D, Lamon S. Circulating microRNAs: let's not waste the potential. Am J Physiol Cell Physiol 2020;2:C313-315. https://doi.org/10.1152/ajpcell.00175.2020

6. Martinez B, Peplow PV. Blood microRNAs as potential diagnostic markers for hemorrhagic stroke. Neural Regen Res 2017;1:13-18. https://doi.org/10.4103/1673-5374.198965

7. Lu M, Dong X, Zhang Z, Li W, Khoshnam SE. Non-coding RNAs in ischemic stroke: roles in the neuroinflammation and cell death. Neurotox Res 2020;38:564-578. https://doi.org/10.1007/s12640-020 -00236-7

8. Li G, Ma Q, Wang R, Fan Z, Tao Z, Liu P, et al. Diagnostic and immunosuppressive potential of elevated mir-424 levels in circulating immune cells of ischemic stroke patients. Aging Dis 2018;9: 172-181. https://doi.org/10.14336/AD.2017.0602

9. Lu WJ, Zeng LL, Wang Y, Zhang Y, Liang HB, Tu XQ, et al. Blood microRNA-15a correlates with IL-6, IGF-1 and acute cerebral ischemia. Curr Neurovasc Res 2018;15:63-71. https://doi.org/10.2174/156 7202615666180319143509

10. Cao W, Zhang T, Wang L, Fu J, Jin H. Diagnostic performance of circulating microRNAs in acute ischemic stroke: A protocol for systematic review and meta-analysis. Medicine 2020;99:40(e22353). https://doi.org/10.1097/MD.0000000000022353

11. Jafarzadeh-Esfehani R, Soudyab M, Parizadeh SM, Jaripoor ME, Nejad PS, Shariati M et al. Circulating exosomes and their role in stroke. Curr Drug Targets. 2020: 21: 89-95. https://doi.org/10.2174/1389 450120666190821153557

12. Kalra H, Drummen GP, Mathivanan S. Focus on extracellular vesicles: Introducing the next small big thing. Int J Mol Sci. 2016; 17: 170. https://doi.org/10.3390/ijms17020170

13. Théry C, Zitvogel L, Amigorena S. Exosomes: composition, biogenesis and function. Nat Rev Immunol. 2002; 2: 569-579. https://doi.org/10.1038/nri855

14. Xia X, Wang Y, Huang Y, Zhang H, Lu H, Zheng JC. Exosomal miRNAs in central nervous system diseases: biomarkers, pathological mediators, protective factors and therapeutic agents. Prog Neurobiol. 2019; 183: 101694. https://doi.org/10.1016/j.pneurobio.2019.101694

15. Yáñez-Mó M, Siljander PR, Andreu Z, Zavec AB, Borràs FE, Buzas EI, et al. Biological properties of extracellular vesicles and their physiological functions. J Extracell Vesicles 2015;4: 27066.

16. Zhang ZG, Chopp M. Exosomes in stroke pathogenesis and therapy. J Clin Invest. 2016;126: 1190-1197. https://doi.org/10.1172/JCI81133

17. Johnsen KB, Gudbergsson JM, Skov MN, Pilgaard L, Moos T, Duroux M. A comprehensive overview of exosomes as drug delivery vehicles - endogenous nanocarriers for targeted cancer therapy. Biochim Biophys Acta. 2014; 1846: 75-87. https://doi.org/10.1016/j.bbcan.2014.04.005

18. Bălașa A, Șerban G, Chinezu R, Hurghiș C, Tămaș F, Manu D. The involvement of exosomes in glioblastoma development, diagnosis, prognosis, and treatment. Brain Sci. 2020: 10: 553. https://doi. org/10.3390/brainscil0080553

19. Kalani A, Tyagi A, Tyagi N. Exosomes: mediators of neurodegeneration, neuroprotection and therapeutics. Mol Neurobiol.2014; 49: 590-600. https://doi.org/10.1007/s12035-013-8544-1

20. Witwer KW, Buzás EI, Bemis LT, Bora A, Lässer C, Lötvall J, et al. Standardization of sample collection, isolation and analysis methods in extracellular vesicle research. J Extracell Vesicles. 2013: 2. https:// doi.org/10.3402/jev.v2i0.20360 
21. Mincheva-Nilsson L, Baranov V, Nagaeva O, Dehlin E. Isolation and characterization of exosomes from cultures of tissue explants and cell lines. Curr Protoc Immunol. 2016; 115: 14.42.1-14.42.21. https://doi.org/10.1002/cpim.17

22. Valadi H, Ekstrom K, Bossios A, Sjostrand M Lee JJ, Lotvall JO. Exosome-mediated transfer of mRNAs and microRNAs is a novel mechanism of genetic exchange between cells. Nat Cell Biol. 2007;9: 654-659. https://doi.org/10.1038/ncb1596

23. Potjewyd G, Moxon S, Wang T, Domingos M, Hooper NM. Tissue engineering 3D neurovascular units: A biomaterials and bioprinting perspective. Trends Biotechnol. 2018; 36: 457-472. https://doi. org/10.1016/j.tibtech.2018.01.003

24. Ozaki T, Nakamura H, Kishima H. Therapeutic strategy against ischemic stroke with the concept of neurovascular unit. Neurochem. Int. 2019; 126: 246-251. https://doi.org/10.1016/j.neuint. 2019.03.022

25. Posada-Duque RA, Barreto GE, Cardona-Gomez GP. Protection after stroke: cellular effectors of neurovascular unit integrity. Front Cell Neurosci. 2014; 8: 231. https://doi.org/10.3389/fncel.2014.00231

26. Steliga A, Kowiański P,Czuba E, Waśkow M, Moryś J, Lietzau G. Neurovascular unit as a source of ischemic stroke biomarkers-limitations of experimental studies and perspectives for clinical application. Transl Stroke Res. 2020; 11: 553-579. https://doi.org/10.1007/s12975-019-00744-5

27. Wang L, Xiong X, Zhang L, Shen J. Neurovascular unit: A critical role in ischemic stroke. CNS Neurosci Ther. 2021;27:7-16. https://doi.org/10.1111/cns.13561

28. Holm MM, Kaiser J, Schwab ME, Extracellular vesicles: Multimodal envoys in neural maintenance and repair. Trends Neurosci. 2018;41:360-372. https://doi.org/10.1016/j.tins.2018.03.006

29. Zagrean AM, Hermann DM, Opris I Zagrean L, Popa-Wagner A. Multicellular crosstalk between exosomes and the neurovascular unit after cerebral ischemia. Therapeutic implications. Front Neurosci. 2018;12:811. https://doi.org/10.3389/fnins.2018.00811

30. Bustamante A, García-Berrocoso T, Rodriguez N, Llombart V, Ribó M, Molina C et al. . Ischemic stroke outcome: A review of the influence of post-stroke complications within the different scenarios of stroke care. Eur J Intern Med. 2016;29:9-21. https://doi.org/10.1016/j.ejim.2015.11.030

31. Ji Q, Ji Y, Peng J, Zhou X, Chen X, Zhao H, et al. Increased brain-specific miR-9 and miR-124 in the serum exosomes of acute ischemic stroke patients. PLoS One 2016, 11, e0163645. https://doi. org/10.1371/journal.pone.0163645

32. Chen Y, Song Y, Huang J, Qu M, Zhang Y, Geng J, et al. Increased circulating exosomal miRNA223 is associated with acute ischemic stroke. Front Neurol. 2017;8:57. https://doi.org/10.3389/ fneur.2017.00057

33. Zhou J, Chen L, Chen B, Huang S, Zeng C, Wu H, et al. Increased serum exosomal miR-134 expression in the acute ischemic stroke patients. BMC Neurol. 2018;18:198. https://doi.org/10.1186/ s12883-018-1196-z

34. Song P, Sun H, Chen H, Wang Y, Zhang, Q. Decreased serum exosomal miR-152-3p contributes to the progression of acute ischemic stroke. Clin Lab. 2020;66. https://doi.org/10.7754/Clin.Lab.2020 200106

35. Wang W, Li DB, Li RY, Zhou X, Yu DJ, Lan XY et al. Diagnosis of hyperacute and acute ischemic stroke: The potential utility of exosomal MicroRNA-21-5p and MicroRNA-30a-5p. Cerebrovasc Dis. 2018; 45:204-212. https://doi.org/10.1159/000488365

36. Li DB, Liu, JL, Wang W, Li RY, Yu DJ, Lan XY, et al. Plasma exosomal miR-422a and miR-125b-2-3p serve as biomarkers for ischemic stroke. Curr Neurovasc Res. 2017,14, 330-337. https://doi.org/10. 2174/1567202614666171005153434

37. Dolz S, Górriz D, Tembl JI, Sánchez D, Fortea G, Parkhutik V et al. Circulating MicroRNAs as novel biomarkers of stenosis progression in asymptomatic carotid stenosis. Stroke 2017;48:10-16. https:// doi.org/10.1161/STROKEAHA.116.013650

38. Couch Y, Akbar N, Davis S, Fischer R, Dickens AM, Neuhaus AA, et al. Inflammatory stroke extracellular vesicles induce macrophage activation. Stroke 2017;48:2292-2296. https://doi.org/10.1161/ STROKEAHA.117.017236

39. Song Y, Li Z, He T, Qu M, Jiang L, Li W et al. M2 microglia-derived exosomes protect the mouse brain from ischemia-reperfusion injury via exosomal miR-124. Theranostics 2019;9:2910-2923. https:// doi.org/10.7150/thno.30879 
40. Yang J, Cao LL, Wang XP, Guo W, Guo RB, Sun YQ, et al. Neuronal extracellular vesicle derived miR98 prevents salvageable neurons from microglial phagocytosis in acute ischemic stroke. Cell Death Dis. 2021;12:23. https://doi.org/10.1038/s41419-020-03310-2

41. Xu B, Zhang Y, Du XF, Li J, Zi HX, Bu JW, et al. Neurons secrete miR-132-containing exosomes to regulate brain vascular integrity. Cell Res. 2017;27:882-897. https://doi.org/10.1038/cr.2017.62

42. Pei X, Li Y, Zhu L, Zhou Z. Astrocyte-derived exosomes suppress autophagy and ameliorate neuronal damage in experimental ischemic stroke. Exp Cell Res. 2019;382:111474. https://doi.org/10.1016/j. yexcr.2019.06.019

43. Pei X, Li Y, Zhu L, Zhou Z. Astrocyte-derived exosomes transfer miR-190b to inhibit oxygen and glucose deprivation induced autophagy and neuronal apoptosis. Cell Cycle 2020;19:906-917. https://doi.org/10.1080/15384101.2020.1731649

44. Chen W, Wang H, Zhu Z, FengJ, Chen L. Exosome-shuttled circSHOC2 from IPASs regulates neuronal autophagy and ameliorates ischemic brain injury via the miR-7670-3p/SIRT1 Axis. Mol Ther Nucleic Acids. 2020;22:657-672. https://doi.org/10.1016/j.omtn.2020.09.027

45. Bu X, Li D, Wang F, Sun Q, Zhang Z. Protective role of astrocyte-derived exosomal microRNA-361 in cerebral ischemic-reperfusion injury by regulating the AMPK/mTOR signaling pathway and targeting CTSB. Neuropsychiatr Dis Treat. 2020;16:574, 1863-1877. https://doi.org/10.2147/NDT.S260748

46. Wu W, Liu J, Yang C, Xu Z, Huang J, Lin J. Astrocyte-derived exosome-transported microRNA-34c is neuroprotective against cerebral ischemia/reperfusion injury via TLR7 and the NF-kB/MAPK pathways. Brain Res Bull. 2020;163:84-94. https://doi.org/10.1016/j.brainresbull.2020.07.013

47. Hira K, Ueno Y, Tanaka R, Miyamoto N, Yamashiro K, Inaba, et al. Astrocyte-derived exosomes treated with a semaphorin 3A inhibitor enhance stroke recovery via prostaglandin D2 synthase. Stroke 2018; 49:580, 2483-2494. https://doi.org/10.1161/STROKEAHA.118.021272

48. Wang S, Cesca F, Loers G, Schweizer M, Buck F, Benfenati F, et al. Synapsin I is an oligomannose carrying glycoprotein, acts as an oligomannose-binding lectin, and promotes neurite outgrowth and neuronal survival when released via glia-derived exosomes. J Neurosci. 2011;31:7275-7290. https:// doi.org/10.1523/JNEUROSCI.6476-10.2011

49. Guitart K, Loers G, Buck F, Bork U, Schachner M, Kleene R. Improvement of neuronal cell survival by astrocyte-derived exosomes under hypoxic and ischemic conditions depends on prion protein. Glia 2016;64:896-910. https://doi.org/10.1002/glia.22963

50. Xu L, Cao H, Xie Y, Zhang Y, Du M, Xu X, et al. Exosome-shuttled miR-92b-3p from ischemic preconditioned astrocytes protects neurons against oxygen and glucose deprivation. Brain Res. 2019;1717:66-73. https://doi.org/10.1016/j.brainres.2019.04.009

51. Li Z, Song Y, He T, Wen R, Li Y, Chen T, et al. M2 microglial small extracellular vesicles reduce glial scar formation via the miR-124/STAT3 pathway after ischemic stroke in mice. Theranostics 2021;11:1232-1248. https://doi.org/10.7150/thno.48761

52. Tian Y, Zhu P, Liu S, Jin Z, Li D, Zhao H, et al. Z. IL-4-polarized BV2 microglia cells promote angiogenesis by secreting exosomes. Adv Clin Exp Med. 2019;28:421-430. https://doi.org/10.17219/ acem/91826

53. Raffaele S, Gelosa P, Bonfanti E, Lombardi M, Castiglioni L, Cimino M, et al. Microglial vesicles improve post-stroke recovery by preventing immune cell senescence and favoring oligodendrogenesis. Mol Ther. 2021;29:1439-1458. https://doi.org/10.1016/j.ymthe.2020.12.009

54. Fröhlich D, Kuo WP, Frühbeis C, Sun JJ, Zehendner CM, Luhmann HJ, et al. Multifaceted effects of oligodendroglial exosomes on neurons: impact on neuronal firing rate, signal transduction and gene regulation. Philos Trans R Soc Lond B Biol Sci. 2014;369:20130510. https://doi.org/10.1098/ rstb.2013.0510

55. Zhou S, Gao B, Sun C, Bai Y, Cheng D, Zhang Y et al. Vascular endothelial cell-derived exosomes protect neural stem cells against ischemia/reperfusion injury. Neuroscience 2020;441:184-196. https://doi.org/10.1016/j.neuroscience.2020.05.046

56. Gao B, Zhou S, Sun C, Cheng D, Zhang Y, Li X, et al. Brain endothelial cell-derived exosomes induce neuroplasticity in rats with ischemia/reperfusion injury. ACS Chem Neurosci. 2020;11:2201-2213. https://doi.org/10.1021/acschemneuro.0c00089 
57. Zhang Y, Qin Y, Chopp M, Li C, Kemper A, Liu X, et . Ischemic cerebral endothelial cell-derived exosomes promote axonal growth. Stroke 2020;51;3701-3712. https://doi.org/10.1161/STROKEAHA. 120.031728

58. Venkat P, Cui C, Chopp M, Zacharek A, Wang F, Landschoot-Ward J, et al. MiR-126 mediates brain endothelial cell exosome treatment-induced neurorestorative effects after stroke in type 2 diabetes mellitus mice. Stroke 2019;50:2865-2874. https://doi.org/10.1161/STROKEAHA.119.025371

59. Wang J, Chen S, Zhang W, Chen Y, Bihl JC. Exosomes from miRNA-126-modified endothelial progenitor cells alleviate brain injury and promote functional recovery after stroke. CNS Neurosci Ther. 2020a;26:1255-1265. https://doi.org/10.1111/cns.13455

60. Wang J, Liu H, Chen S, Zhang W, Chen Y, Yang Y. Moderate exercise has beneficial effects on mouse ischemic stroke by enhancing the functions of circulating endothelial progenitor cell-derived exosomes. Exp Neurol. 2020b;330:113325. https://doi.org/10.1016/j.expneurol.2020.113325

61. Xiao B, Chai Y, Lv S, Ye M, Wu M, Xie L, et al. Endothelial cell-derived exosomes protect SH-SY5Y nerve cells against ischemia/reperfusion injury. Int J Mol Med. 2017;40:1201-1209. https://doi.org/ 10.3892/ijmm.2017.3106

62. Aldewachi H, Al-Zidan EN, Conner MT, Salman MM. High-throughput screening platforms in the discovery of novel drugs for neurodegenerative diseases. Bioengineering (Basel) 2021;8:30. https:// doi.org/10.3390/bioengineering8020030

63. Salman MM, Al-Obaidi Z, Kitchen P, Loreto A, Bill RM, Wade-Martins R. Advances in applying computer-aided drug design for neurodegenerative diseases. Int J Mol Sci. 2021;22:4688. https://doi. org/10.3390/ijms22094688

64. Wevers NR, Kasi DG, Gray T, Wilschut KJ, Smith B, van Vught R, et al. A perfused human blood-brain barrier on-a-chip for high-throughput assessment of barrier function and antibody transport. Fluids Barriers CNS. 2018;15:23. https://doi.org/10.1186/s12987-018-0108-3

65. Salman MM, Marsh G, Kusters I, Delincé M, Di Caprio G, Upadhyayula S, et al. Design and validation of a human brain endothelial microvessel-on-a-chip open microfluidic model enabling advanced optical imaging. Front Bioeng Biotechnol. 2020;8:573775. https://doi.org/10.3389/fbioe.2020.573775

66. Nzou G, Wicks RT, VanOstrand NR, Mekky GA, Seale SA, El-Taibany A,et al. Multicellular 3D neurovascular unit model for assessing hypoxia and neuroinflammation induced blood-brain barrier dysfunction. Sci Rep. 2020;10:9766. https://doi.org/10.1038/s41598-020-77348-9

67. Wang SN, Wang Z, Xu TY, Cheng MH, Li WL, Miao CY. Cerebral organoids repair ischemic stroke brain injury. Transl Stroke Res. 2020;11:983-1000. https://doi.org/10.1007/s12975-019-00773-0

68. Song H, Zhang X, Chen R, Miao J, Wang L, Cui L, et al. Cortical neuron-derived exosomal microRNA181c-3p inhibits neuroinflammation by down regulating CXCL1 in astrocytes of a rat model with ischemic brain injury. Neuroimmunomodulation 2019;26:217-233. https://doi.org/10.1159/000502694

69. Pala E, Bustamante A, Jolkkonen J, Hommel M, Rosell A, Montaner J. Blood-based biomarkers and stem cell therapy in human stroke: a systematic review. Mol Biol Rep 2020;47:6247-58. https://doi. org/10.1007/s11033-020-05627-9

70. Ghafouri-Fard S, Shoorei H, Taheri M. Non-coding RNAs participate in the ischemia-reperfusion injury. Biomed Pharmacother 2020;110419. https://doi.org/10.1016/j.biopha.2020.110419

71. Zhang ZG, Buller B, Choop M. Exosomes - beyond stem cells for restorative therapy in stroke and neurological injury Nat Rev Neurol 2019;15:193-203. https://doi.org/10.1038/s41582-018-0126-4

72. Venkat P, Choop M. Exosome treatment for stroke with diabetic comorbidity. Neural Regen Res 2022;17:315-317. https://doi.org/10.4103/1673-5374.319190 
